\title{
Relation Analysis of Taiwan and Japan Stock Markets with the Factors of U.S. and U.K. Stock Markets
}

\author{
CHING-HUEI CHEN \\ Department of Hospital and Health Care Administration, Chia Nan University of Pharmacy \& Science, \\ No. 60, Erh-Jen Rd., Sec.1, Jen-Te, Tainan, 71710, Taiwan. \\ Email: wilsonchen0831@yahoo.com.tw \\ Tel: +886-6-2664911 ext.5208 \\ WANN-JYI HORNG \\ Department of Hospital and Health Care Administration, Chia Nan University of Pharmacy \& Science, \\ No. 60, Erh-Jen RD., Sec.1, Jen-Te, Tainan, Taiwan. \\ Email: hwj7902@mail.cnu.edu.tw \\ Tel: +886-6-2664911 ext.5220
}

\begin{abstract}
Evaluating the relationship of the two stock markets, in this paper, the evidence results show that the proposed model is appropriate for Taiwan and Japanese stock markets. The evidence result also indicates that the two study market is a positive relation. The average estimation value of correlation coefficient equals to 0.408, which implies that the two study markets, is synchronized influence. On the other hand, the study result also shows that the two stock markets do not have asymmetrical effect. The study result also indicates that U.K. and U.S. stock return rate volatilities affects the return rate volatilities of Taiwanese stock market, and U.K. and U.S. stock return rate volatilities also affects the return rate volatilities of Japanese stock market. Besides, U.S. stock return volatility rates also truly affects the variation risks of two study markets.
\end{abstract}

Keywords: Stock return, Asymmetric Effect, IGARCH Model.

\section{Introduction}

According to UNCTAD's World Investment Report 2013, by the end of 2012. Taiwan's cumulative foreign investment amounted to $\$ 226.093$ billion, an increase of $6.1 \%$ over 2011 , ranking 21 st in the world, fifth in Asia, next to Hong Kong, Japan, Mainland China and Singapore. Taiwan has become a major foreign investor in Asia, with a global investment mix. Besides, its growth of economy in 2014 is $4.0 \%$. Apparently, Taiwan plays an important role in the global economic financial system. We also know that, Japan and Taiwan have the relationships of the trade and the tour. And Japan is the second largest economy country after the United States, with gross domestic product (GDP) reaching \$497.2 billion in 2003, which is about half the total of Asian countries; ranked fourth in the world after Norway, the United States and Switzerland; and total trade accounted for $5.6 \%$ of the world, second only to the United States and Germany, ranked third.

As the investor has an investment in the international stock market, he/she will usually care about the international capital the motion situation, the international politics and the economic situation change, in particular, in the U.K. and the U.S. stock markets change. There is a relationship for Taiwan and Japan 
based on the trade and the circulation of capital with the U.K. and the U.S., but the U.K. and U.S. are also powerful global economic nations. Therefore, the relationship between the Taiwan and the Japanese stock markets are worth further discussion.

The purpose of the study paper is to examine the relations of the Taiwan and the Japanese stock markets. Study paper also further discusses the influence of the U.S. and the U.K. stock return rates' volatility for the Taiwan and the Japanese stock markets.

The organization of this paper is as follows: Section 2 descibes the data characteristics; Section 3 introduces the asymmetric test of the DCC and the bivariate $\operatorname{IGARCH}(1,2)$; Section 4 presents the proposed model and the evidence results, and finally Section 5 summarizes the conclusions of this study.

\section{Stock Data Characteristics}

\section{Study Data Sources}

The data sources of this research included the U.K., the U.S., the Taiwan and the Japanese stock price collected between January, 2005 and December, 2013. The source of the stock data was the Taiwan economic Journal (TEJ), a database in Taiwan.

The U.K. stock price refers to the FTSE100 index, the U.S. stock price refers to the S\&P500 index. The Taiwan stock price refers to the Taiwan weighted stock price index, the Japanese stock price refers to Tokyo NK-225 index. The study samples of four stock markets are 1,999.

\section{Return Rate Calculation and Statistics}

The return rate of Japan stock price is calculated by the natural logarithm difference, rides 100 again. The return rates of Taiwan, U.K. and U.S. stock prices are also calculated the natural logarithm difference, rides 100 again. In Table 1, the kurtosis coefficients of the four sequences are all bigger than 3 , and the normal distribution test of Jarque-Bera is not normal distribution. Therefore, the bivariate student t distribution of heavy tail is used in this paper.

Table 1. Study data statistics

\begin{tabular}{|c|c|c|c|c|}
\hline Statistics & RTW & RJAPAN & RUK & RUS \\
\hline Mean & -0.0323 & -0.0382 & -0.0209 & -0.02226 \\
\hline S-D & 1.7522 & 1.6494 & 1.3708 & 1.4191 \\
\hline Kurtosis & 6.6155 & 8.1845 & 9.1357 & 10.3685 \\
\hline J-B N & $1132.62^{\text {**** }}$ & $2320.29^{* * * *}$ & $3135.28^{\text {**** }}$ & $4521.83^{\text {**** }}$ \\
(p-value) & $(0.0000)$ & $(0.0000)$ & $(0.0000)$ & $(0.0000)$ \\
\hline sample & 1998 & 1998 & 1998 & 1998 \\
\hline
\end{tabular}

Notes: (1) S-D is denoted the standard deviation. (2) J-B N is the normal distribution test of Jarque-Bera.

$(3)^{* *}$ denote significance at the level $5 \%$ and ${ }^{* * *}$ denote significance at the level $1 \%$.

\section{KSS Unit Root Test}

The KSS (Kapetanios et al., 2003) unit root test method is used to determine the stability of the time series data. The unit root test results are listed in Table 2. Ithe test result shows that the four stock return rates do not have the characteristics of unit root, this is, the four stock market data are stationary series data, under $\alpha=1 \%$ and $\alpha=5 \%$ significance level. 
Table 2. Unit root test of KSS for the return rate data

\begin{tabular}{|lllll|}
\hline \hline & RTW & RJA & RUK & RUS \\
\hline \hline Statistic & $-19.35^{* * * *}$ & $-20.21^{* * *}$ & $-20.36^{* * *}$ & $-26.03^{* * * *}$ \\
\hline Critical value & -2.82 & -2.22 & -1.92 & \\
\hline Significant level & $\alpha=1 \%$ & $\alpha=5 \%$ & $\alpha=10 \%$ & \\
\hline \hline
\end{tabular}

$\begin{aligned} \text { Notes: } & { }^{* *} \text { denote significance at the level } 5 \% \text { and } \\ & \text { denote significance at the level } 1 \% .\end{aligned}$

\section{Johansen's Co-integration Test}

Johansen's (1991) co-integration test method is used to determine co-integration of four stock markets. The test results are listed in Table 3 at the significance level of 0.05 ( $\alpha=5 \%)$ does not reveal of $\lambda_{\text {max }}$ statistic. This indicated that the four stock markets do not have a co-integration relation. Therefore, we do not need to consider the model of error correction.

Table 3. The results of Co-integration test (VAR lag=7)

\begin{tabular}{|lll|}
\hline \hline$H_{0}$ & $\lambda_{\max }$ & Critical value \\
\hline \hline None & 14.3806 & 30.8151 \\
\hline At most 1 & 13.5255 & 24.2520 \\
\hline At most 2 & 4.9824 & 17.1477 \\
\hline At most 3 & 2.9138 & 3.8415 \\
\hline \hline
\end{tabular}

Notes: The lag of VAR is selected by the AIC rule (Akaike, 1973).

The critical value is given under the level $5 \%$.

\section{ARCH Effect Test}

By the proposed model as below, we uses the methods of LM test (Engle, 1982) and F test (Tsay, 2004) to test the conditionally heteroskedasticity phenomenon. In Table 4, the test results of the ARCH effect show that the two markets have the conditionally heteroskedasticity phenomenon exists. Study result suggests that we can use the GARCH model to match and analyze it.

Table 4. ARCH effect test

\begin{tabular}{|ccc|}
\hline \hline TW & Engle LM test & Tsay F test \\
\hline \hline Statistic & $422.285^{* * * *}$ & $6.438^{* * *}$ \\
\hline (p-value) & $(0.0000)$ & $(0.0000)$ \\
\hline JA & Engle LM test & Tsay F test \\
\hline \hline Statistic & $671.969^{* * *}$ & $21.274^{* * *}$ \\
\hline (p-value) & $(0.0000)$ & $(0.0000)$ \\
\hline
\end{tabular}

Notes : ${ }^{* *}$ denote significance at the level $5 \%$ and ${ }^{* * *}$ denote significance at the level $1 \%$.

\section{Asymmetric Test of the Proposed Model}

The proposed model in Table 5 will be used to determine the asymmetric. It can be constructed in this paper, it is stated as below. The test methods of Engle and $\mathrm{Ng}$ (1993) are used as the negative size bias test and joint test.

By the negative size bias and the joint test results shows that the two study markets do not have the asymmetrical effect in Table 5. 
Table 5. Asymmetric test of the DCC and the bivariate-IGARCH $(1,2)$

\begin{tabular}{|ccc|}
\hline \hline JA & Joint test & Negative size bias test \\
\hline \hline F statistic & 1.1530 & 0.0375 \\
\hline (p-value) & $(0.3264)$ & $(0.7863)$ \\
\hline \hline TW & Joint test & Negative size biast test \\
\hline \hline F statistic & 1.5279 & 1.4340 \\
\hline (p-value) & $(0.2053)$ & $(0.2313)$ \\
\hline \hline
\end{tabular}

Notes: (1) ${ }^{* *}$ denote significance at the level $5 \%$ and ${ }^{* * *}$ denote significance at the level $1 \%$.

(2) p-value $<\alpha$ denote significance. ( $\alpha=1 \%, \alpha=5 \%, \alpha=10 \%)$.

\section{Proposed Model and Evidence Results}

Based on the results of the asymmetric test as above, we follows the ideas of the papers of Ding, Granger and Engle (1993) and Ding and Engle (2001). By the model selection, in this paper, we may use the DCC and $\operatorname{GARCH}(1,2)$ model to construct the two study markets. The proposed model is illustrated as follows:

$$
\begin{aligned}
& R T W_{t}=\phi_{0}+\phi_{1} R T W_{t-1}+\phi_{2} R J A_{t-1}+\phi_{3} R U S_{t-1}+\phi_{4} R U K_{t-1}+a_{1, t}, \\
& R J A_{t}=\varphi_{0}+\varphi_{1} R J A_{t-1}+\varphi_{2} R T W_{t-1}+\varphi_{3} R U S A_{t-1}+\varphi_{4} R U K_{t-1}+a_{2, t}, \\
& h_{11, t}=c_{10}+c_{11} a_{1, t-1}^{2}+c_{12} a_{1, t-2}^{2}+d_{11} h_{11, t-1}+\eta_{1} R U S_{t-2}^{2}, \\
& h_{22, t}=c_{20}+c_{22} a_{2, t-2}^{2}+d_{21} h_{22, t-1}+\eta_{2} R U S_{t-2}^{2}, \\
& h_{12, t}=r_{t} \sqrt{h_{11, t}} \sqrt{h_{22, t}}, \\
& r_{t}=\exp \left(q_{t}\right) /\left(\exp \left(q_{t}\right)+1\right), \\
& q_{t}=\gamma_{0}+\gamma_{1} \rho_{t-1}+\gamma_{2} a_{1, t-1} a_{2, t-1} / \sqrt{h_{11, t-1} h_{22, t-1}},
\end{aligned}
$$

with the white noise of $\vec{a}_{t}^{\prime}=\left(a_{1, t}, a_{2, t}\right)$ is obey the bivariate Student's $t$ distribution, this is,

$$
\vec{a}_{t} \sim T_{v}\left(\overrightarrow{0},(v-2) H_{t} / v\right),
$$

the $r_{t}$ is the DCC coefficient of $a_{1, t}$ and $a_{2, t}$, the bivariate distribution details can refer the book of Tsay (2004). The maximum likelihood algorithm method of BHHH (Berndt et. al., 1974) is used to estimate the model's unknown parameters. The soft programs of EVIEWS and RATS are used in this paper.

By the evidence results, we know that the two study markets may be constructed on the DCC and the bivariate IGARCH $(1,2)$ model. Its estimate result is stated in Table 6.

The evidence results show that the Taiwan's stock return rate volatility receives before 1 period's influence of the U.S. stock return rate volatility ( $\phi_{3}=0.3456$ ). The Taiwan's stock return rate volatility also receives before 1 period's influence of the U.K. stock return rate volatility $\left(\phi_{4}=0.0568\right)$. The Taiwan stock return rate volatility does not also receive before 1 period's influence of the Taiwan and Japan stock return rate volatility.

The Japanese stock return rate volatility receives before 1 period's influence of the Japanese stock return rate volatility $\left(\varphi_{1}=-0.0943\right)$. The Japanese stock return rate volatility also receives before 1 period's 
influence of the Taiwan stock return rate volatility $\left(\varphi_{2}=-0.0356\right)$. The Japanese stock return rate volatility also receives before 1 period's influence of the U.S. stock return rate volatility $\left(\varphi_{3}=0.4265\right)$. The Japanese stock return rate volatility also receives before 1 period's influence of the U.K. stock return rate volatility ( $\varphi_{4}=0.1643$ ). The stock return rate volatilities of the U.S. and the U.K. are truly influent the return rate volatilities of the two stock markets.

Besides, the correlation coefficient (average estimation value $\bar{r}_{t}=0.408$ ) of the two stock return rate volatility is significant. This result also shows the two stock return rate volatilities are mutually synchronized influence. On the other hand, estimated value of the degree of freedom for the Student's t distribution is 7.18, and is significant under the significance level of $0.01(\alpha=1 \%)$. This also demonstrates that this study data has the heavy tailed distribution.

The estimated coefficient values of the conditional variance equation products the different variation risks in the two stock markets in Table 6. The square item (lag=2) of the U.S. stock return rates also affects the variation risk of the two stock markets ( $\eta_{1}=0.0644$ and $\eta_{2}=0.0371$, respectively). We have also the result of $c_{11}+c_{12}+d_{11}+\eta_{1}=1$ in the Taiwanese stock market, and $c_{22}+d_{21}+\eta_{2}=1$ in the Japanese stock market. This result conforms the conditionally supposition of the IGARCH model. The evidence results also show that the two stock markets are the IGARCH model. This result also demonstrates the proposed model may catch the two stock market volatilities' process. The evidence result also shows that the Taiwan stock market has the fixed variation risk, the Japanese stock market has also a fixed variation risk. In Table 6 , the two stock markets have the different conditional variation risks. The variation risk of the Taiwan is larger than the variation risk of Japan's stock market.

Table 6. The results of Parameter estimation for the proposed model

\begin{tabular}{|llcccc|}
\hline \hline Parameters & $\phi_{0}$ & $\phi_{1}$ & $\phi_{2}$ & $\phi_{3}$ & $\phi_{4}$ \\
\hline Coefficient value & 0.0474 & -0.0074 & -0.0039 & 0.3456 & 0.0568 \\
\hline (p-value) & $(0.0723)$ & $(0.7337)$ & $(0.8580)$ & $(0.0000)$ & $(0.0715)$ \\
\hline Parameters & $\varphi_{0}$ & $\varphi_{1}$ & $\varphi_{2}$ & $\varphi_{3}$ & $\varphi_{4}$ \\
\hline Coefficient value & 0.0219 & -0.0943 & -0.0356 & 0.4265 & 0.1643 \\
\hline (p-value) & $(0.3686)$ & $(0.0000)$ & $(0.0532)$ & $(0.0000)$ & $(0.0000)$ \\
\hline Parameters & $c_{10}$ & $c_{11}$ & $c_{12}$ & $d_{11}$ & $\eta_{1}$ \\
\hline Coefficient value & 0.0195 & 0.0056 & 0.0206 & 0.9094 & 0.0644 \\
\hline (p-value) & $(0.0003)$ & $(0.6791)$ & $(0.0004)$ & $(0.0004)$ & $(0.0001)$ \\
\hline Parameters & $c_{20}$ & $c_{22}$ & $d_{21}$ & $\eta_{2}$ & \\
\hline \hline Coefficient value & 0.0230 & 0.0838 & 0.8791 & 0.0371 & \\
\hline (p-value) & $(0.0003)$ & $(0.0000)$ & $(0.0000)$ & $(0.0000)$ & \\
\hline Parameters & $\gamma_{0}$ & $\gamma_{1}$ & $\gamma_{2}$ & $\bar{r}_{t}$ & $v$ \\
\hline \hline Coefficient value & -2.1098 & 4.1355 & 0.0834 & 0.4076 & 7.1812 \\
\hline (p-value) & $(0.0000)$ & $(0.0000)$ & $(0.0002)$ & $(0.0000)$ & $(0.0000)$ \\
\hline Parameters & $\min r_{t}$ & $\max r_{t}$ & & & \\
\hline \hline Coefficient value & 0.2031 & 0.8721 & & & \\
\hline (p-value) & \multicolumn{5}{l}{}
\end{tabular}

Notes: p-value $<\alpha$ denote significance. $(\alpha=1 \%, \alpha=5 \%, \alpha=10 \%)$. 
The autocorrelation of the standard residual error may test for the proposed model. The details are omitted in this paper. The test result shows that proposed model does not show an autocorrelation of the standard residual error. The proposed model as above are more appropriate.

\section{Conclusions}

The two study markets may construct in the proposed model with the factors of the U.K. and the U.S. stock markets. The evidence results show that the two stock markets do not have the asymmetric. The evidence result also obtains that the DCC coefficient estimation value $\left(\bar{r}_{t}=0.408\right)$ of the two stock markets, and the relation of two stock markets is positive. The U.K. and the U.S. stock return rate volatilities truly affects the stock return rate volatilities of the Taiwan stock market. The variation risks of the two stock markets are also truly received the impact of the U.S. stock return rate volatilities.

\section{References}

Akaike, H. (1973). Information theory and an extension of the maximum likelihood principle, In 2 nd. International Symposium on Information Theory, edited by B. N. Petrov and F. C. Budapest: Akademiai Kiado, 267-281.

Berndt, E.K., Hall, B.H., Hall, R.E. and Hausman, J.A. (1974)..Estimation and inference in nonlinear structural models. Annals of Economic and Social Measurement, 4, 653-665.

Ding, Z. and Engle, R. F. (2001). Large scale conditional covariance matrix modeling, estimation and testing. Academia Economic Papers, 29, 157-184.

Ding, Z., Granger, C.W.J. and ngle, R.F. (1993). A long memory property of stock market returns and a new model. Journal of Empirical Finance, 1, 83-106.

Engle, R.F. (1982). Autoregressive conditional heteroscedasticity with estimates of the variance of United Kingdom Inflation. Econometrica, 50, 987-1007.

Engle, R.F. and Ng, V.K. (1993). Measuring and Testing the Impact of News on Volatility, Journal of Finance. 48(5), 1749-1777.

Johansen, S. (1991). Estimation and Hypothesis Testing of Cointegration Vector in Gaussian Vector Autoregressive Models. Econometrica, 52, 389-402.

Kapetanios, G., Shin, Y. and Snell, A. (2003). Testing for a unit root in the nonlinear STAR framework. Journal of Econometrics, 112(2), 359-379.

Nelson, D.B. (1991). Conditional heteroscedasticity in asset returns: A new Approach. Econometrica, 59, 347-370.

Tsay, R.S. (2004). Analysis of Financial Time Series. New York: John Wiley \& Sons, Inc. 